\title{
Evaluation of osteonectin as a diagnostic marker of osteogenic bone tumors
}

\author{
Murad Ahmad ${ }^{1}$, Sadaf Mirza ${ }^{2}$, Kafil Akhtar,", Rana K. Sherwani ${ }^{4}$, Khalid A Sherwani ${ }^{5}$ \\ ${ }^{1}$ Assistant Professor, ${ }^{2}$ Resident, ${ }^{3,4}$ Professor, ${ }^{1-4}$ Dept. of Pathology, Jawaharlal Nehru Medical College, Aligarh Muslim \\ University, Aligarh, Uttar Pradesh, ${ }^{5}$ Professor, Dept. of Orthopaedics Surgery, Jawaharlal Nehru Medical College, Aligarh \\ Muslim University, Aligarh, Uttar Pradesh, India
}

*Corresponding Author:

Email: drkafilakhtar@gmail.com

\begin{abstract}
Introduction: Immunohistochemistry plays an important but limited role in the diagnosis of primary bone tumors. Sometimes it is very difficult to differentiate osteosarcomas histologically from other tumors with similar morphology but with different malignant potential and treatment protocol. The correct diagnosis of OSA relies on identification of osteoid production by malignant cells which can be detected by the use of osteonectin.

Materials and Methods: The present study was carried out on 200 patients of benign and malignant lesions of bone. After a detailed clinical history and local examination, paraffin section of resected specimens were studied by hematoxylin and eosin and immunohistochemical stain, osteonectin and matrix was graded on a four-tiered grading system with statistical analysis of osteonectin positivity in osteogenic bone tumours and tumour-like lesions.

Results: Benign and malignant tumours accounted for $74.6 \%$ and $25.0 \%$ of the total cases. Osteoid production were seen in 36 cases (100.0\%) of osteosarcomas, followed by fibrous dysplasia in 18 cases (100.0\%) and osteoid osteoma in 10 cases (100.0\%). Both polygonal and spindle shaped tumour cells in osteosarcoma showed Grade 4 positivity with osteonectin. Fibroblastic variant of osteosarcoma showed diffuse (Grade 4) and focal strong staining (Grade 2) alternating with negative areas. Chondroblastic variant of osteosarcoma showed Grade 4+ immunoreactivity in conventional osteosarcoma like areas, whereas chondrocyte- like tumour cells surrounded by chondro-osteoid -like matrix showed well- defined osteonectin staining.

Conclusions: Osteonectin is a helpful osteogenic marker for establishing the diagnosis of osteoblastic origin of a tumour that produce no or scarcely any matrix.
\end{abstract}

Keywords: Bone Tumors, Immunohistochemistry, Osteonectin.

\section{Introduction}

Bone tumours represents only $1.7 \%$ of orthopaedic lesions. Osteosarcoma is the most common type of bone cancer; a malignant mesenchymal tumour in which the cancerous cells produce bone matrix. It occurs in all age groups but has a bimodal age distribution. Seventy- five percent occur in person younger than 20 years, with male preponderance. It is also common over 60 years of age. ${ }^{1}$

Immunohistochemistry plays an important but limited role in the diagnosis of primary bone tumors. The results should be carefully assessed taking into account the clinical and radiological features of the case and the morphology on the haematoxylin and eosin stained sections. The prognostic value of antibodies such as the proliferation marker MIB1 and markers for multiple drug resistance, may in future, become part of the standard panel for some primary bone tumors.

SPARC (secreted protein, acidic and rich in cysteine), also known as osteonectin or BM-40 (basement membrane tumor matrix component), is a secreted calcium-binding glycoprotein. ${ }^{2}$ Rather than acting as structural components of the extracellular matrix, members of this family mediate cell-matrix interactions. ${ }^{3,4}$

Sometimes it is very difficult to differentiate osteosarcomas histologically from other tumors with similar morphology but with different malignant potential and treatment protocol. Therefore the correct diagnosis is necessary for a better outcome. The correct diagnosis of OSA relies on identification of osteoid production by malignant cells which can be detected by the use of osteonectin.

\section{Materials and Methods}

The present study was carried out on 200 patients of benign and malignant lesions of bone, attending the Outpatients and Inpatients Departments of Orthopaedics Surgery and Pathology, Jawaharlal Nehru Medical College, Aligarh Muslim University, Aligarh for a period of 5 years.

A detailed history and a thorough clinical examination, including local examination of bony lesions was carried out along with relevant investigations like histopathology of paraffin embedded section of specimens using hematoxylin and eosin stain. Histologically proven benign and malignant lesions were selected for the study. Subsequently, serial sections, were used for the immunohistochemical analysis, using rabbit antihuman polyclonal antibodies. Mesenchymal tumours without bone formation like Fibrosarcoma, Rhabdomyosarcoma, Leiomyosacoma and Ewing's sarcoma served as controls.

Osteonectin immunostaining of tumor cells and matrix was graded on a four-tiered grading system: $1=$ focal $(<50 \%)$ weak staining; $2=$ focal strong staining; 3 = diffuse (>50\%) weak staining; 4 = diffuse strong staining. Statistical Analysis of osteonectin positivity in 
osteogenic bone tumours and tumour-like lesions was also calculated

\section{Observations}

Most of the patients in this study had benign tumours, accounting for $74.6 \%$ of the cases whereas malignant tumours accounted for $25.0 \%$ of the total cases.
Most common tumour was giant cell tumour, which accounted for $45(22.5 \%)$ cases, followed by osteosarcoma 36 cases (18.0\%), Fibrous dysplasia 18 cases $(9.0 \%)$, Aneursymal bone cyst 15 cases $(7.5 \%)$, osteochondroma 14 cases $(7.0 \%)$, callus formation 14 cases $(7.0 \%)$, osteoid osteoma 10 cases $(5.0 \%)$, chondrosarcoma 9 cases $(4.5 \%)$, enchondroma 8 cases (4.0\%). (Table 1)

Table 1: Distribution of different types of tumours according to who classification

\begin{tabular}{|l|c|c|}
\hline \multicolumn{1}{|c|}{ Types of tumours } & No. of cases & Percentage \\
\hline A. Cartilage forming tumours & & \\
\hline Osteochondroma & 14 & 7.0 \\
\hline Enchondroma & 08 & 4.0 \\
\hline Chondromyxoid fibroma & 02 & 1.0 \\
\hline Chondroblastoma & 02 & 1.0 \\
\hline Chondrosarcoma & 09 & 4.5 \\
\hline Dedifferentiated tumours & & 1.0 \\
\hline B. Bone forming tumours & 10 & \\
\hline Osteoid osteoma & 01 & 5.0 \\
\hline Osteoblastoma & 36 & 0.5 \\
\hline Osteosarcoma & & 18.0 \\
\hline C. Fibrogenic tumours & 02 & 1.0 \\
\hline Fibrosarcoma & & \\
\hline D. Primitive Neuroecto-Dermal & 08 & 4.0 \\
\hline Ewing's sarcoma & 45 & 22.5 \\
\hline E. Giant Cell Tumour & & \\
\hline F. Notochordal & 03 & 1.5 \\
\hline Chordoma & & \\
\hline G. Muscle & 02 & 1.0 \\
\hline Rhabdomyosarcoma & 03 & 1.5 \\
\hline Leiomyosarcoma & & \\
\hline H. Miscellaneous & 18 & 9.0 \\
\hline Fibrous dysplasia & 15 & 7.5 \\
\hline Aneursymal bone cyst & 14 & 7.0 \\
\hline Callus formation & 03 & 1.5 \\
\hline Simple bone cyst & $\mathbf{2 0 0}$ & 1.5 \\
\hline Non- ossifying fibroma & $\mathbf{1 0 0}$ \\
\hline Total & & \\
\hline
\end{tabular}

In our study, maximum number of cases of osteoid production were seen in osteosarcomas, 36 cases $(100.0 \%)$, followed by fibrous dysplasia, 18 cases
(100.0\%), osteoid osteoma, 10 cases (100.0\%), 10 cases $(71.0 \%)$ each of callus formation and osteochondroma and 9 cases $(60.0 \%)$ of aneurysmal bone cyst. (Table 2)

Table 2: Distribution of different tumours and tumour- like lesions with osteoid production

\begin{tabular}{|l|c|c|c|}
\hline Tumour and tumour like lesions & No. of cases & $\begin{array}{c}\text { Osteoid } \\
\text { present }\end{array}$ & Osteoid absent \\
\hline Osteosarcoma & 36 & 36 & - \\
\hline Chondrosarcoma & 09 & - & 09 \\
\hline Callus formation & 14 & 10 & 04 \\
\hline Fibrous dysplasia & 18 & 18 & - \\
\hline Giant cell tumour & 45 & - & 45 \\
\hline Aneurysmal bone cyst & 15 & 09 & 07 \\
\hline Osteoid osteoma & 10 & 10 & - \\
\hline Osteochondroma & 14 & 10 & 04 \\
\hline Osteoblastoma & 01 & - & 01 \\
\hline
\end{tabular}




\begin{tabular}{|l|c|c|c|}
\hline Chondromyxoid fibroma & 02 & & 02 \\
\hline Enchondroma & 08 & & 08 \\
\hline Chondroblastoma & 02 & - & 02 \\
\hline Dedifferentiated tumours & 02 & & 02 \\
\hline Fibrosarcoma & 02 & - & 02 \\
\hline Ewing sarcoma & 08 & - & 08 \\
\hline Leiomyosarcoma & 03 & - & 03 \\
\hline Rhabdomyosarcoma & 02 & - & 02 \\
\hline Simple bone cyst & 03 & - & 03 \\
\hline Non- ossifying fibroma & 03 & & 03 \\
\hline Chordoma & 03 & - & 03 \\
\hline Total & $\mathbf{2 0 0}$ & $\mathbf{9 3}$ & $\mathbf{1 0 7}$ \\
\hline
\end{tabular}

Two $(1.0 \%)$ cases of rhabdomyosarcoma were seen, with pleomorphic rhabdomyoblasts with multinucleation and osteonectin negativity in the malignant tumour cells. Eight (4\%) cases of Ewing's sarcoma was seen, with clusters of small round cells with hyperchromatic nucleus with mild karyomegaly and scant cytoplasm, infiltrating the bone. All the soft tissue tumours of similar morphological structures (fibrosarcoma, leiomyosarcoma, rhabdomyosarcoma and Ewing's sarcoma) were negative for osteonectin, but Ewing's sarcoma showed CD99 positivity.

Our results showed that all cases of osteosarcoma $(100 \%)$ reacted with the osteonectin antibody. A positive immunohistochemical reaction was not only found in the osteoblastic variant, but also in the fibrosarcomatous and pleomorphic variants of the osteosarcoma. Nine cases of Osteoblastic osteosarcoma showed predominantly thick anastomosing lace-like pink osteoid formation by malignant tumour cells having high N/C ratio and marked nuclear pleomorphism, with focal areas of mineralization (Fig. 1). Both polygonal and spindle shaped tumour cells showed Grade 4 positivity with osteonectin (Fig. 2). Poorly mineralized matrix was weakly stained (Grade 1 positivity). (Tables 3 and 4 )

Table 3: Distribution of bone tumour and tumours like lesions showing osteonectin positivity

\begin{tabular}{|l|c|c|c|}
\hline \multirow{2}{*}{ Tumour Type } & \multirow{2}{*}{ No. of cases } & \multicolumn{2}{|c|}{ Osteonectin Positivity } \\
\cline { 3 - 4 } & & No. of positive cases & Percentage \\
\hline Osteosarcoma & 36 & 36 & 100 \\
\hline Osteoblastic & 9 & 09 & 100 \\
\hline Fibroblastic & 03 & 03 & 100 \\
\hline Chondroblastic & 04 & 04 & 100 \\
\hline Telangiectatic & 03 & 03 & 100 \\
\hline Small cell & 03 & 03 & 100 \\
\hline Giant cell rich & 04 & 04 & 100 \\
\hline Anaplastic & 02 & 02 & 100 \\
\hline Epithelioid & 03 & 03 & 100 \\
\hline Periosteal & 03 & 03 & 100 \\
\hline Chondrosarcoma & 09 & - & -- \\
\hline Enchondroma & 08 & - & - \\
\hline Chondromyxoid fibroma & 02 & - & - \\
\hline Chondroblastoma & 02 & 02 & 100 \\
\hline Differentiated cartilagenous tumours & 02 & - & - \\
\hline Giant cell tumours & 45 & - & - \\
\hline Osteoid osteoma & 10 & 10 & 100 \\
\hline Fibrous dysplasia & 18 & 18 & 100 \\
\hline Aneuysmal bone cyst & 15 & 06 & 40.0 \\
\hline Callus formation & 14 & 10 & 66.6 \\
\hline Osteoblastoma & 01 & 01 & 100 \\
\hline Osteochondroma & 14 & 11 & 78.5 \\
\hline Chordoma & 03 & - & - \\
\hline Simple bone cyst & 03 & - & - \\
\hline Non-ossifying fibroma & 03 & - & - \\
\hline Ewing's sarcoma & 08 & - & - \\
\hline
\end{tabular}




\begin{tabular}{|l|l|l|l|}
\hline Rhabdomyosarcoma & 02 & - & - \\
\hline Leiomyosarcoma & 03 & - & - \\
\hline Fibrosarcoma & 02 & - & - \\
\hline
\end{tabular}

Table 4: Intensity of the immunohistochemical reaction of osteonectin antibodies in different types of bone lesions

\begin{tabular}{|c|c|c|c|c|c|c|}
\hline \multirow[t]{2}{*}{ Types of bone tumours } & \multirow[t]{2}{*}{$\begin{array}{l}\text { Total No. of } \\
\text { cases }\end{array}$} & \multicolumn{5}{|c|}{$\begin{array}{l}\text { No. of cases with degree of immunoreactivity in the } \\
\text { tumour cells }\end{array}$} \\
\hline & & $\mathbf{0}$ & $1+$ & $2+$ & $3+$ & $4+$ \\
\hline Osteoblastic osteosarcoma & 09 & - & 01 & 01 & - & 07 \\
\hline Fibroblastic osteosarcoma & 03 & - & - & 01 & - & 02 \\
\hline Chondroblastic Osteosarcoma & 04 & - & - & - & - & 04 \\
\hline Telangiectatic Osteosarcoma & 02 & - & - & - & - & 02 \\
\hline Small cell osteosarcoma & 04 & - & - & - & - & 04 \\
\hline Giant cell rich osteosarcoma & 04 & - & - & - & - & 04 \\
\hline Anaplastic Osteosarcoma & 02 & - & - & - & - & 02 \\
\hline Epithelioid Osteosarcoma & 03 & - & - & - & 03 & - \\
\hline Low grade osteosarcoma & 02 & - & - & 02 & - & - \\
\hline Periosteal Osteosarcoma & 03 & - & - & - & 03 & - \\
\hline Fibrous dysplasia & 18 & - & $01^{\wedge}$ & 02 & 05 & 10 \\
\hline Aneurysmal bone cyst & 15 & - & 03 & 03 & - & - \\
\hline Osteoid osteomas & 10 & - & - & 04 & - & 06 \\
\hline Bengin giant cell tumours & 45 & - & - & - & - & - \\
\hline Callus formation & 14 & - & - & 04 & 10 & - \\
\hline Osteochondroma & 14 & - & - & 09 & 03 & - \\
\hline Osteoblastoma & 01 & - & - & 01 & - & - \\
\hline Chondroblastoma & 02 & - & - & 01 & - & 01 \\
\hline Enchondroma & 08 & - & - & - & - & - \\
\hline Chondromyxoid fibroma & 02 & - & - & - & - & - \\
\hline Simple bone cyst & 02 & - & - & - & - & - \\
\hline Non- ossifying fibroma & 02 & - & - & - & - & - \\
\hline Chordoma & 03 & - & - & - & - & - \\
\hline Ewing's sarcoma & 08 & - & - & - & - & - \\
\hline Rhabdomyosarcoma & 02 & - & - & - & - & - \\
\hline Leiomyosarcoma & 03 & - & - & - & - & - \\
\hline Fibrosarcoma & 02 & - & - & - & - & - \\
\hline
\end{tabular}

Three cases of Fibroblastic variant of osteosarcoma had proliferating spindle fibroblastic cells with moderate amount of osseous-matrix without cartilage. It showed diffuse (Grade 4) and focal strong staining (Grade 2) alternating with negative areas (Fig. 3).

Two cases of Anaplastic variant of osteosarcoma had microscopic appearance of high grade conventional osteosarcoma with tumour cells showing marked pleomorphism, increased N/C ratio, hyperchromatic nuclei and marked osteoid production. Spindle cells as well as multinucleated malignant giant cells showed strong and diffuse immunoreactivity with osteonectin (Grade 4).

Four cases of Chondroblastic variant of osteosarcoma showed undifferentiated stromal cells, lobules of malignant cartilage admixed with foci of osteoid and woven bone. Degree of immunoreactivity was Grade 4+ in conventional osteosarcoma like areas, whereas chondrocyte- like tumour cells surrounded by chondro-osteoid -like matrix showed well- defined osteonectin staining (Fig. 4). This indicates a switch over of cellular metabolism leading to the production of bone matrix protein, similar to the process of enchondral ossification. This switch- over can be explained by the known fact that a combination of cartilage specific type II collagen and bone typical I collagen occurs in chondroid bone. Chondro-osteoid and intimately associated cells (chondroid bone) were positive for osteonectin antibody. Chondro-osteoid showed intense immunoreactivity in focal areas of chondrosarcoma as well as chondroblastic osteosarcoma.

Three cases of Epithelioid Osteosarcoma showed tumour cells with minimal pleomorphism, high N/C ratio, hyperchromatic nuclei with abundant cytoplasm and epithelioid differentiation and malignant osteoid production. Diffuse weak staining (Grade 3) of osteonectin was observed on IHC in malignant cells 
with plasmacytoid features. Myogenin for rhabdomyosarcoma and CD38 and CD138 for Multiple Myeloma and smooth muscle actin (SMA) was negative in cases of epithelioid osteosarcoma.

Small cell variant of osteosarcoma on microscopy showed very small round to ovoid malignant cells with hyperchromatic nuclei and minimal osseous matrix production. Diffuse strong staining (Grade 4) was observed in the malignant cells. Small amount of osteoid present showed focal weak staining (Grade 1).

Four cases of Giant cell rich osteosarcoma showed microscopic features of conventional osteosarcoma along with formation of malignant giant cells with osseous matrix production.

Three cases of Periosteal variant of osteosarcoma showed lobules of malignant cartilage, permeating the pre-existing trabeculae of bone and the chondroblastic centre of the lobules of cartilage showed osseousmatrix formation.

Three cases of Telangiectatic variant of osteosarcoma showed cystic areas with blood and solid areas of neoplastic spindle cells with multiple areas of osteoid production. Undifferentiated spindle cells within the wall of the cystic area also showed evidence of osteoid production.

Nine cases of Chondrosarcoma showed microscopic features of well differentiated hyaline cartilage, exhibiting foci of hypercellularity and moderate nuclear atypia without direct osteoid or bone formation by the tumour cells. The malignant chondroid mesenchymal cells were negative for osteonectin.

Forty-five cases of Giant cell tumours comprised of uniformly distributed multinucleated giant cells and round to oval atypical mononuclear stromal cells, which showed no immunoreactivity with antiosteonectin antibody. However reactive bone (a common finding) showed diffuse positive reaction (Grade 2) in osteoblasts rimming the newly formed trabeculae. The same features were also observed in the reactive bone lesion (callus) in morphologically similar cells which were located in the neighbourhood of newly formed trabeculae.

Aneurysmal bone cyst was microscopically composed of blood filled spaces separated by septa consisting of loosely arranged spindle cells along with benign giant cells, which were osteonectin negative.

Chondroblastoma showed mixture of mononuclear cells and giant cells. Mononuclear cells had oval nucleoli with longitudinal groove, with pink cytoplasm with distinct cytoplasmic outline. Chondroid differentiation was seen in the matrix with focal areas of calcification. The chondromatous tumours were negative for osteonectin but presented a special feature: Single positively reacting cells (Grade 2 to Grade 4 positivity) located near the mineralization zones were seen in chondroblastoma that consisted of fibroblastic spindle shaped cells. The immediately adjacent multinucleated osteoclastic giant cells were negative. Other chondroid tumors like enchondroma and chondromyxoid fibroma also showed negative immunoreactivity for osteonectin

Osteoid osteoma histologically showed a well circumscribed lesion with central nidus formation, consisting of anastomozing bony trabeculae rimmed by osteoblast with variable degree of mineralization. Strong osteonectin immunoreactivity was apparent in osteoblasts and osteogenic stroma, with minimal staining in the bone matrix. All cuboidal, osteoblastic cells around the newly formed trabeculae revealed intense cytoplasmic staining (Grade 4). Positive reaction was also observed in oval to spindle cells located centrally in the nidus area. Extracellular matrix showed minimal staining with osteonectin antibodies.

Fibrous dysplasia showed narrow and misshaped bony trabeculae interspersed with fibrous tissue; lining osteoblasts was not present on the surface of the trabeculae. Eighteen cases (100\%) of fibrous dysplasia that we studied showed a distinctly positive reaction (Grade 1) with the osteonectin antibodies, chiefly in cells immediately adjacent to newly formed woven bone or cells located within the trabeculae of woven bone. The reaction differed in degree, with few cases showing diffuse positivity covering the whole cytoplasm and some with focal positivity with granular intracytoplasmic distribution. The spindle shaped cells on the surface of woven bone showed intense diffuse positivity (Grade 4).

Osteochondroma microscopically revealed mature bony trabeculae covered by a cartilaginous cap composed of normal hyaline cartilage. Osteonectin antibody was apparently positive in the osteoblastic cells rimming the trabeculae as well as cells within the bony trabeculae in 11 out of the 14 cases studied.

In our study, the cytoplasmic staining with osteonectin showed $90.9 \%$ sensitivity and $66.6 \%$ specificity in the identification of tumours with osteoblastic differentiation. (Table 5)

Table 5: Correlation of osteonectin marker and histopathological evaluation

\begin{tabular}{|c|c|c|c|c|}
\hline \multirow{4}{*}{ Osteonectin } & & \multicolumn{3}{c|}{ Histopathological Findings } \\
\cline { 2 - 5 } & & Positive & Negative & Total \\
\cline { 2 - 5 } & Positive & 100 & 30 & 130 \\
\cline { 2 - 5 } & Negative & 10 & 60 & 70 \\
\hline \multicolumn{2}{|c|}{ Total } & 110 & 90 & 200 \\
\hline
\end{tabular}




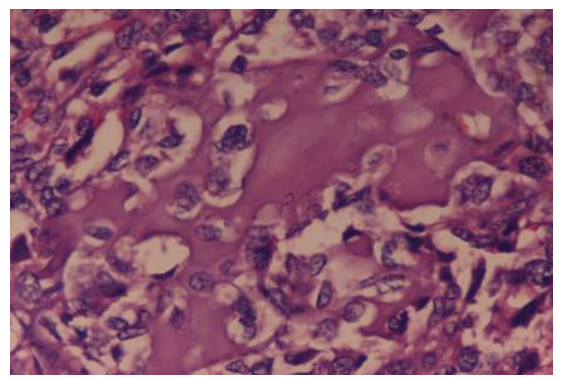

Fig. 1: Osteoblastic osteosarcoma: Photomicrograph shows thick lace like pink osteoid with malignant tumor cells with high $\mathrm{N} / \mathrm{C}$ ratio and marked pleomorphism. Haematoxylin and eosin $x$ 40X.

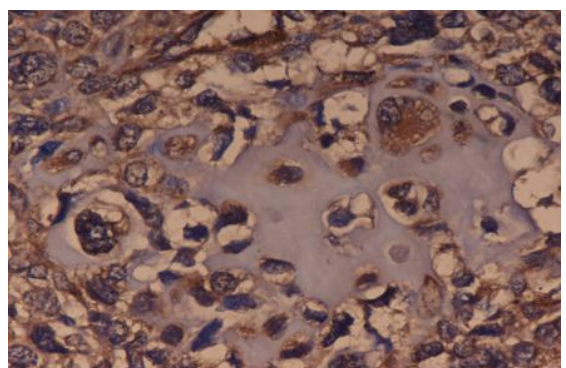

Fig. 2: Osteoblastic osteosarcoma: Photomicrograph shows osteonectin positivity in malignant cells. immunostain osteonectin $x$ 40X.

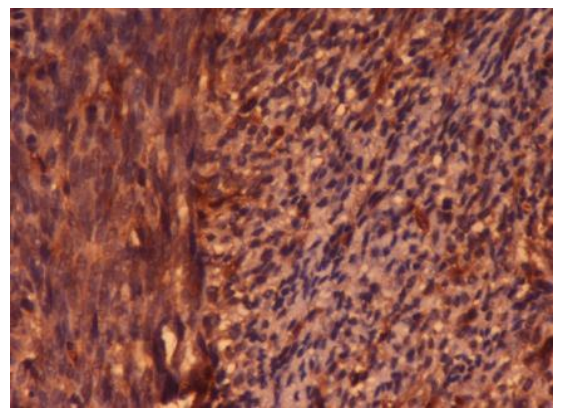

Fig. 3: Fibroblastic Osteosarcoma:

Photomicrograph shows intense cytoplasmic staining in malignant spindle cells (Grade 4), alternating with lighter areas (Grade 2). immunostain osteonectin $\mathrm{x} 10 \mathrm{X}$.

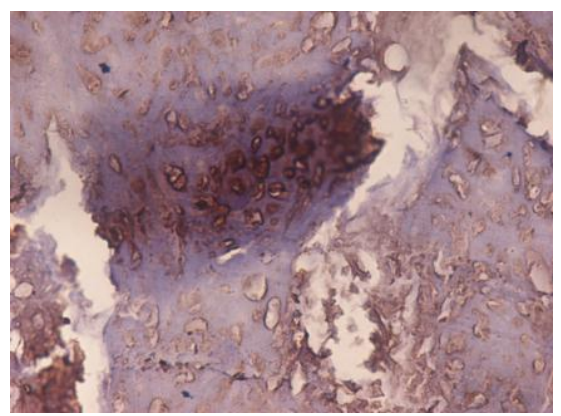

Fig. 4: Chondroblastic osteosarcoma:

Photomicrograph shows chondrocyte-like tumor cells surrounded by chondro-osteoid like matrix, with well-defined osteonectin marking. immunostain osteonectin $\mathrm{x}$ 40X.

\section{Discussion}

Osteoblasts differ from other connective tissue cells by their ability to form bone matrix. Approximate $90 \%$ of the total protein of bone consists of type I collagen. Although this is the only collagen type present in bone, it cannot be regarded as a bone-specific marker because it is also found in other tissues. ${ }^{5}$ The specific properties of bone are therefore modified by its other, non-collagenous protein components. One of these proteins is osteonectin. It possesses a high affinity for hydroxyapatite and type I collagen, and one of its functions may be to connect the two molecules. ${ }^{6}$

Previous immunohistochemical studies have shown that osteonectin is a marker for the differentiation of bone matrix forming cells (osteoblasts). ${ }^{7}$ From these results, the question arises as to whether osteonectin is also produced by the cells of bone tumours, and whether it might be used as a marker for this tumour group? To, elucidate this question, the present immunohistochemistry study was performed to determine the presence of osteonectin in benign and malignant bone tumours.

The demonstration of osteonectin in bone tumours have proved to be a valuable factor in the diagnosis of bone-forming tumours, in particular of osteosarcoma. In combination with the light microscopic findings, a positive osteonectin reaction makes it possible for the pathologist to classify a tumour as an osteosarcoma, even if only a small amount of material is available. The identification of osteonectin in the fibroblastic and anaplastic types of osteosarcoma is of special importance because it can prove that these cells belonged histogenetically to the osteosarcoma tissue. Same is true for small cell osteosarcoma, which may simulate Ewing's sarcoma histologically. The production of osteonectin depends on the functional state of an osteoblast. The intensity of the immunohistochemical reaction differed and showed a clear- cut quantitative dependence on the differentiation of the tumour tissue. ${ }^{8}$

Diagnosis becomes difficult in a cases of osteosarcoma, if the tumour shows no matrix production, or if matrix production is scanty and hard to recognize. In such cases, the immunohistochemical demonstration of osteonectin would be an important and helpful tool for establishing the definite diagnosis of osteosarcoma.

In our study, we found that in osteoblastic osteosarcomas, both polygonal and spindle shaped tumour cells showed diffuse strong positivity with osteonectin and poorly mineralized matrix was weakly stained. In fibroblastic osteosarcoma, two patterns were identified: diffuse strong staining and focal strong staining, alternating with negative areas. Schultz et al in 1988 , found that all the subtypes of osteosarcomas reacted with osteonectin antibody due to the presence of osteonectin. ${ }^{9}$ In cases of anaplastic osteosarcoma, spindle cells as well as multinucleated malignant giant 
cells showed strong and diffuse immunoreactivity with osteonectin, findings concordant with the study by Schultz et al in $1988 .^{9}$

Osteonectin is absent in all non-skeletal tissues with the exception of chondrocytes in so called mineralizing chondrocytes. ${ }^{10}$ Osteonectin is formed by cells which do not yet posses the morphological phenotype of osteoblasts and may regarded as a "diffentiation marker"of the osteoblastic lineage. Only chondroid bone (tissue in which chondrocytes surrounded by osteoid matrix containing type 1 and type 2 collagen) showed a positive reaction. All other primary skeletal tumours and extraskeletal soft tissue tumours were completely negative. ${ }^{11}$

In our study, benign lesions like osteoid osteoma also showed strong osteonectin immunoreactivity in the osteoblasts and the osteogenic stroma, with minimal staining in the bone matrix. The osteoid osteoma nidus showed intense cytoplasmic staining in cuboidal, osteoblastic cells around the newly formed trabeculae. This finding was consistent with Jundt et al 1989, who reported 3 cases of osteoid osteoma with positive osteonectin antibodies. $^{11}$

All cases of fibrous dysplasia showed a positive immunoreactivity with osteonectin antibody in our study. The intensity of osteonectin reactivity in our study decreased with the distance of the cells from the woven bone. Jundt et al in 1989 have also reported a distinctly positive reaction with the osteonectin antibodies in all cases of fibrous dysplasia, whereas Sakamoto, in 1999 found only a single case out of twenty cases of fibrous dysplasia with positive osteonectin immunoreactivity. ${ }^{11,12}$

The chondromatous tumours were negative for osteonectin but presented a special feature: single positively immunoreacting cells located near the mineralization zones were seen in chondroblastoma that consisted of fibroblastic spindle shaped cells (Grade 2 to Grade 4 positivity). But Fanburg et al in 1999, found cellular areas and centrally in the obvious chondroid areas were invariably osteonectin negative, but two cases of chondroblastoma showed osteonectin positivity in their study showed osteonectin positivity in myxoid spindle cell areas and peripherally in the chondroid areas. The adjacent multinucleated osteoclastic giant cells were negative for osteonectin antibody. ${ }^{13}$

In our study chondrosarcomas showed negative reaction to osteonectin antibodies. Quite similarly a study done by Jundt et al in 1989 and Dahlin \& Unni in 1986, reported that malignant mesenchymal cells in chondrosarcomas were osteonectin negative, as was the chondroid matrix. However, cartilage with a positive cytoplasmic reaction was observed in the matrix zones that displayed an osteoid- like staining behavior under the light microscope. ${ }^{11,14}$ This pattern of immunoreactivity was also noted in the chondromatous portions of the osteosarcomas. ${ }^{11}$ Bosse et al, 1990, have reported that positive reactions appeared only in the vicinity of trabeculae and in dedifferentiated areas in chondrosarcomas. ${ }^{15}$

Multinucleated (osteoclast) giant cells and mononuclear spindle cells in giant cell tumours showed no immunoreactivity with antiosteonectin antibody. However reactive bone showed diffuse positivity reaction in the osteoblasts rimming the newly formed trabeculae in our study, findings which correlated well with the reports by Jundt et al, 1989. ${ }^{11}$ The same features has been observed in callus of newly formed trabeculae, a finding concordant with the study by Meng in 1999, who found 15 cases of callus formation to be immunoreactive for osteonectin antibody, with focal strong positivity. ${ }^{16}$

Multinucleated giant cells in aneurysmal bone cysts and chondroblastoma showed no immunoreactivity with osteonectin in our study, a finding concordant with the study by Jundt et al, 1989.

In our study, the cytoplasmic staining with osteonectin showed $90.9 \%$ sensitivity and $66.6 \%$ specificity in the identification of tumours with osteoblastic differentiation. This was correlated well with the study done by Fanburg et al 1997, who found $90.0 \%$ sensitivity and $54.0 \%$ specificity of osteonectin immunoreactivity in bone-forming tumors. ${ }^{13}$

\section{Conclusions}

Osteonectin antibodies react reliably with benign and malignant bone-forming tumours and may be regarded as a "differentiation marker" of the osteoblastic lineage. The correct diagnosis of osteosarcoma relies on identification of malignant osteoblasts that are capable of producing neoplastic bone. Osteonectin positivity in tumour cells and osteoblasts around chondroid bone can help in differentiating osteosarcoma from other non-boneforming sarcomas, particularly in differentiating small cell osteosarcoma from other small round cell tumours. Immunohistochemical demonstration of osteonectin is an important denominator and a helpful osteogenic marker for establishing the diagnosis of osteoblastic origin of a tumour that produce no or scarcely any matrix.

\section{References}

1. Klein MJ and Siegal GP. Osteosarcoma anatomic and histologic variants. Am J Clin Pathol 2006; 125: 555-556.

2. Yan Q and Sage EH. SPARC- a matricellular glycoprotein with important biological functions. $J$ Histochem Cytochem 1999;47 (12):1495-1506.

3. Bornstein P. Diversity of function is inherent in matricellular proteins: an appraisal of thrombospondin 1 . J Cell Biol 1995;130(3):503-506.

4. Brekken RA and Sage EH. SPARC, a matricellular protein: at the crossroads of cell-matrix communication. Matrix Biol 2001;19(8):816-827.

5. Von der Mark K. Localization of collagen types in tissues. Int Rev Connect Tissue Res 1981; 9:265-324. 
6. Termine JD, Kleinman HK, Whitson SW, Conn KM, McGarvey ML, Martin GR. Osteonectin, a bone-specific protein linking mineral to collagen. Cell 1981;26(1):99105.

7. Jundt G, Berghäuser KH, Termine JD, Schulz A. Osteonectin-a differentiation marker of bone cells. Cell Tissue Res 1987;248(2):409-415.

8. Schultz A, Fischer HP, Breithaupt H, Pralle H. TherapieResponse verschiedener histologischer Subtypendes Osteosarkoms unter hochdosierter Methotrexatbehandlung (therapeutic response of various histological subtypes of osteosarcoma following treatment with high dosages of methotrexate). Onkologie 1983;6:296-304

9. Schulz A, Jundt G, Berghauser KH, Gehron-Robey P, Termine JD. Immunohistochemical Study of Osteonectin in Various Types of Osteosarcoma; Am J Pathol 1988;132(2):1-5.

10. Jundt G, Berghäuser KH, Termine JD, Schulz A. Osteonectin-a differentiation marker of bone cells. Cell Tissue Res 1987;248(2):409-415.

11. Jundt G, Schulz A, Berghäuser KH, Fisher LW, GehronRobey P, Termine JD. Immunocytochemical identification of osteogenic bone tumors by osteonectin antibodies. Virchows Arch A Pathol Anat Histopathol 1989;414(4):345-353.

12. Sakamoto A, Oda Y, Iwamoto Y, Tsuneyoshi M. A comparative study of fibrous dysplasia and osteofibrous dysplasia with regard to expressions of c-fos and c-jun products and bone matrix proteins: a clinicopathologic review and immunohistochemical study of c-fos, c-jun, type I collagen, osteonectin, osteopontin, and osteocalcin. Hum Pathol 1999;30(12):1418-1426.

13. Fanburg JC, Rosenberg AE, Weaver DL, Leslie KO, Mann KG, Tracy RP. Osteocalcin and osteonectin immunoreactivity in the diagnosis of osteosarcoma. Am J Clin Pathol 1997;108(4):464-473.

14. Dahlin DC and Unni KK. Bone Tumors. General Aspects and data on 8,542 cases, $4^{\text {th }}$ Edn. Springfield, IL, Charles C Thomas 1986;pp 141-148.

15. Bosse A, Vollmer E, Bocker W, Rossener A. The impact of osteonectin for differential diagnosis of bone tumors: An immunohistochemical approach. Pathol Res Practice 1990;186(5):651-657.

16. Meng G, Ottaviani G, Jaffe N. The epidemiology of Ewing's Sarcoma and Osteosarcoma. Cancer Treat Res 2009;152:3-13. 